medgen $2011 \cdot 23: 437-437$

DOI 10.1007/s11825-011-0299-3

Online publiziert: 12. Dezember 2011

(c) Springer-Verlag 2011

\author{
M. Speicher ${ }^{1}$ B. Eiben ${ }^{2}$ \\ ${ }^{1}$ Institut für Humangenetik der Medizinischen Universität Graz \\ ${ }^{2}$ MVZ Institut für Labormedizin und Klinische Genetik Rhein/Ruhr GmbH, Essen
}

\title{
Pränatal-, Präimplantations- und Polkörperdiagnostik
}

tung für verschiedene Facharztgruppen. Es folgen 2 Beiträge, die sich mit etablierten nichtinvasiven pränatalen Verfahren befassen, nämlich der Sonographie und dem Ersttrimesterscreening. Beide Methoden werden weitgehend außerhalb der Humangenetik durchgeführt. Der vierte Beitrag beschäftigt sich mit der Amniozentese und Chorionzottenbiopsie und im anschließenden fünften Beitrag werden die Möglichkeiten der molekularzytogenetischen Methoden und Array-Diagnostik in der Pränatalmedizin beschrieben. Die Beiträge sechs und sieben setzen sich mit der Präimplantationsdiagnostik für monogene Erkrankungen und der Polkörperdiagnostik auseinander. Der letzte Beitrag geht auf die neuen Verfahren ein, die durch das sog. „next generation sequencing" möglich wurden und die ein hohes Potenzial haben, die pränatale Diagnostik grundlegend zu verändern.

Diese neuen technischen Möglichkeiten sind gleichzeitig mit zunehmenden, schwierig zu beantwortenden ethischen Problemen verbunden. Diese ethische Problematik wurde erst kürzlich in Deutschland bei der sehr kontroversen Diskussion um die Zulassung der Präimplantationsdiagnostik im Deutschen Bundestag wieder offenbar. Die neuen Möglichkeiten der nichtinvasiven diagnostischen Verfahren, insbesondere durch die neuen Sequenziertechniken, bieten zwar den Vorteil, dass der Fetus durch die Untersuchung an sich nicht gefährdet wird, erlauben aber auch nicht nur quantitative Analysen, sondern auch Aussagen über Mutationen bei der Mutter und potenziell auch den Mutationsstatus des Vaters bis hin zur Möglichkeit, eine ge- nomweite Karte des Mutationsstatus des Feten aus maternaler DNA zu erstellen, wie dies bereits in einer ersten Arbeit [1] gezeigt werden konnte.

Ist alles, was machbar ist, wirklich wünschenswert? Durch die wissenschaftlich technische Innovation entstehen ethischen Fragen, die weit über den Umfang der bisherigen Diskussionen hinausgehen, was pränatal getestet werden sollte und welche Informationen an die werdenden Eltern weitergegeben werden dürfen. Wir müssen uns rechtzeitig mit diesen Möglichkeiten auseinandersetzen, um nicht von der faktischen Entwicklung überrollt zu werden. Wie in der Stellungnahme der deutschen Gesellschaft für Humangenetik zur Zulassung der Präimplantationsdiagnostik vom 01.09.2011 gefordert wurde, erscheint es heute zunehmend wichtiger, dass diese neuen Entwicklungen von einer interdisziplinär besetzten Ethikkommission auch mit Expertinnen und Experten aus den entsprechenden humangenetischen Fachgesellschaften permanent begleitet werden.

\section{Prof. Dr. M. Speicher \\ Prof. Dr. B. Eiben}

\section{Literatur}

1. Lo YM, Chan KC, Sun H, Chen EZ, Jiang P, Lun FM, Zheng YW, Leung TY, Lau TK, Cantor CR, Chiu RW (2010) Maternal plasma DNA sequencing reveals the genome-wide genetic and mutational profile of the fetus. Sci Transl Med 2:61ra91

Die wissenschaftliche Koordination des vorliegenden Themenschwerpunkts erfolgte unter der Leitung von Prof. Dr. med. Michael Speicher, Graz, und Prof. Dr. rer. nat. Bernd Eiben, Essen. 